\title{
Development and characterisation of a bath-based vertical blackbody cavity calibration source for the range $-30^{\circ} \mathrm{C}$ to $150{ }^{\circ} \mathrm{C}$
}

\author{
Sam Boles \\ National Standards Authority Ireland (NSAI) \\ Igor Pušnik \\ University of Ljubljana \\ Dubhaltach Mac Lochlainn \\ National Standards Authority Ireland (NSAI)
}

See next page for additional authors

Follow this and additional works at: https://arrow.tudublin.ie/cieoart

Part of the Atomic, Molecular and Optical Physics Commons, Electrical and Computer Engineering Commons, and the Optics Commons

\section{Recommended Citation}

Sam Boles, Igor Pušnik, Dubhaltach Mac Lochlainn, David Fleming, Izabela Naydenova, Suzanne Martin, Development and characterisation of a bath-based vertical blackbody cavity calibration source for the range $-30^{\circ} \mathrm{C}$ to $150^{\circ} \mathrm{C}$, Measurement, Volume 106, 2017, Pages 121-127, ISSN 0263-2241, DOI: 10.1016/ j.measurement.2017.03.023.

This Article is brought to you for free and open access by the Centre for Industrial and Engineering Optics at ARROW@TU Dublin. It has been accepted for inclusion in Articles by an authorized administrator of ARROW@TU Dublin. For more information, please contact arrow.admin@tudublin.ie, aisling.coyne@tudublin.ie, gerard.connolly@tudublin.ie.

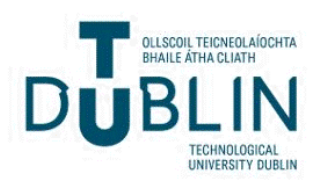




\section{Authors}

Sam Boles, Igor Pušnik, Dubhaltach Mac Lochlainn, David Fleming, Izabela Naydenova, and Suzanne Martin 
See discussions, stats, and author profiles for this publication at: https://www.researchgate.net/publication/314487403

\section{Development and Characterisation of a Bath-Based Vertical Blackbody Cavity Calibration Source for the Range $-30^{\circ} \mathrm{C}$ to $150{ }^{\circ} \mathrm{C}$}

Article $\cdot$ March 2017

Dol: 10.1016/j.measurement.2017.03.023

\section{CITATIONS}

6

6 authors, including:

Sam Boles

National Standards Authority of Ireland

5 PUBLICATIONS 6 CITATIONS

SEE PROFILE

2. Dubhaltach Mac Lochlainn

12. NSAI National Metrology Laboratory

15 PUBlicationS 61 CITATIONS

SEE PROFILE
491

Igor Pušnik

University of Ljubljana

83 PUBLICATIONS 407 CITATIONS

SEE PROFILE

Izabela Naydenova

Technological University Dublin - City Campus

170 PUBLICATIONS 2,500 CITATIONS

SEE PROFILE

Some of the authors of this publication are also working on these related projects:

Investigation of Photopolymer-based Holographic Optical Elements for Solar Applications View project

Development of holographic sensors for monitoring relative humidity and temperature View project 


\title{
Development and Characterisation of a Bath-Based Vertical Blackbody Cavity Calibration Source for the Range $-30{ }^{\circ} \mathrm{C}$ to $150{ }^{\circ} \mathrm{C}$
}

\author{
Sam Boles ${ }^{\text {a, b, }}{ }^{*}$, Igor Pušnik ${ }^{c}$, Dubhaltach Mac Lochlainn ${ }^{a}$, David Fleming ${ }^{\text {a }}$, Izabela Naydenova ${ }^{\text {b }}$, Suzanne Martin ${ }^{\text {b }}$ \\ ${ }^{a}$ National Standards Authority Ireland (NSAI), Dublin, Ireland \\ ${ }^{b}$ Centre for Industrial and Engineering Optics, School of Physics, Dublin Institute of Technology, Dublin, Ireland \\ ${ }^{\mathrm{c}}$ Metrology Institute of the Republic of Slovenia/University of Ljubljana-Faculty of Electrical \\ Engineering/Laboratory of Metrology and Quality (MIRS/UL-FE/LMK), Ljubljana, Slovenia
}

\section{ABSTRACT}

Industrial use of Radiation Thermometers (RTs) is becoming increasingly common due to the perceived advantages and wide market availability. Blackbody Cavity Radiation Sources (BCRSs) are typically used for calibration of these instruments, and these cavities are oriented horizontally in most cases. For BCRSs based in thermal baths, this necessitates the use of custom-built baths with side openings. This paper presents a unique design of vertical bathbased BCRS that may be immersed in conventional calibration baths without modifications to the baths. The method, results, and analysis of an international comparison comparing this vertical BCRS, standard horizontal BCRSs, and a previous iteration of the vertical design of BCRS are also presented. The comparison was conducted through collaboration between the Laboratory of Metrology and Quality, Slovenia (LMK) and the National Standards Authority Ireland (NSAI), with the intention of evaluating the suitability of the vertical orientation for calibration work. Transfer pyrometers and Standard Platinum Resistance Thermometers (SPRTs) were used as comparison standards. The transfer pyrometers used have spectral sensitivity from $8 \mu \mathrm{m}$ to $14 \mu \mathrm{m}$ in this temperature range. It was found that the vertical orientation was comparable to within $0.25{ }^{\circ} \mathrm{C}$ throughout the range to standard horizontal cavities. It was concluded that a vertical configuration is an economical alternative for calibration of RTs within the range assessed.
ARTICLE INFO

Article history:

Received 30 July 2016

Received in revised form 7 March 2017

Accepted 8 March 2017

Keywords:

Blackbody cavity

Radiation thermometer

Blackbody orientation

Infrared thermometer

Calibration

\section{Introduction}

Thermometric traceability to the International Temperature Scale of 1990 (ITS-90) is becoming a more and more common requirement for Radiation Thermometers (RTs). The principal method of linking radiation temperature measurements to contact methods is through the use of blackbody radiation sources of known emissivity. The temperature of these blackbody sources may be monitored by using conventional Standard Platinum Resistance Thermometers (SPRTs) and thermocouples in thermal contact with the source, or by using a radiation thermometer. In either case, instruments with traceability to ITS-90 are used.

Emissivity - a dimensionless quantity with values ranging from zero, for perfectly reflective surfaces, to unity for bodies that absorb all incident radiation - is a key parameter in radiation thermometry [1]. The performance of a practical BCRS is measured in terms of its spectral emissivity, $\varepsilon_{\text {cavity, }}$ which is a ratio comparing the spectral radiance of the real body to that of an ideal blackbody, having an emissivity of unity. In order to achieve a low calibration uncertainty when calibrating a RT, a blackbody of the highest possible overall emissivity should be used. To realize such a blackbody source with a total emissivity close to unity, a blackbody cavity should have a high temperature uniformity and an opening much smaller than the cavity volume [2].

Numerous designs of BCRS exist that fulfil these requirements, utilising stirred baths, heat pipes and fixed points (phase transitions of certain high-purity materials). Cylindrical and conical shapes are most commonly used, as they can ensure a high temperature uniformity along the cavity's length. Bath-based horizontal BCRSs have been demonstrated as robust sources for the calibration of RTs from $-50{ }^{\circ} \mathrm{C}$ up to $300{ }^{\circ} \mathrm{C}[3,4]$. Vertically oriented immersible BCRSs are used for the calibration of tympanic thermometers [5] and sometimes for ice-point checks of industrial RTs [6].

The principal advantage of using a removable immersible vertical cavity is flexibility. The BCRS may be immersed in different baths containing various temperature media. This enables the use of conventional calibration baths already in widespread use in temperature calibration labs, thus significantly reducing the cost of establishing in-house traceability of RTs to contact methods over the cavity's temperature range.

Another potential advantage of the vertical orientation relates to its use below the ambient dew point temperature. Horizontal cavities have a limitation at low temperatures due to the build-up of dew or ice on the internal walls. Typically purging with an inert gas is required to reduce this effect. In the vertical orientation, it has been observed that there is comparably less dew or ice formation, possibly due to stagnation of the air within the cavity. Vertical cavities covering temperatures above and below those used for tympanic thermometers seem to have been largely avoided, presumably due to expected problems with convection at high temperatures, although after an extensive literature survey, no data was available to support this.

The present work was undertaken to investigate if a unique lowcost design of vertical BCRS, constructed in-house to take advantage of equipment already in-situ at NSAl, was suitable for calibration use over the temperature range from $-30{ }^{\circ} \mathrm{C}$ to $150{ }^{\circ} \mathrm{C}$. A reference RT was used to compare vertical and horizontal cavities in order to determine whether measurements from the two orientations were in agreement, once their associated uncertainties were accounted for. The two RTs used in the comparison, which were both manufactured by Heitronics

* Corresponding author at: National Standards Authority of Ireland (NSAI), Dublin, Ireland. Tel.: +35318082674

E-mail: Sam.Boles@NSAl.ie (S. Boles) 
(models TRT II and TRT IV.82), allowed measurements with an uncertainty ranging from $0.34{ }^{\circ} \mathrm{C}$ to $0.66{ }^{\circ} \mathrm{C}(k=2)$ to be made over the temperature range of interest.

Three of the horizontal cavities used for the comparison are part of the suite of BCRSs in LMK, the national temperature standards laboratory for Slovenia. The newly-constructed NSAI vertical cavity was also compared to a similar vertical cavity that had previously been constructed at LMK. In addition, measurements were made to compare the new vertical NSAI cavity against the horizontal BCRSs in the Heitronics calibration laboratory, which is ISO 17025 accredited.

The results reported should facilitate the establishment of measurement capabilities for the calibration of RTs in NSAI and other National Metrology Institutes (NMIs).

\section{Materials and Methods}

\subsection{BCRS Design considerations}

Many RTs cover temperature ranges that span over $1000{ }^{\circ} \mathrm{C}$. It is difficult to achieve good stability and uniformity within calibration media, even in contact thermometry, over such a wide range. Blackbody sources provide improved uncertainty and may be designed and constructed more practically when optimised over narrower temperature ranges and so an array of blackbodies is usually required. Using an array of blackbodies has other advantages like being able to calibrate at multiple temperatures simultaneously.

Two key design considerations for a practical and versatile BCRS are:

- the temperature range the cavity will cover; and
- the emissivity settings and optical characteristics of the RTs that the cavity will be used to calibrate.

RTs commonly have a limited number of emissivity settings. These settings are intended to correspond to the surface emissivity of common materials with the most commonly available setting being 0.95. If using a BCRS with an unmatched emissivity, corrections must be applied to account for this difference [7].

\subsubsection{Filling the Field of View (FOV)}

For a blackbody source to be of practical use for calibrating a given $\mathrm{RT}$, the opening must be at least large enough to allow a RT's FOV to be filled. RT manufacturers typically specify the FOV as a ratio between the target distance and the diameter of the RT's measuring spot (spot size) at that distance, but it may also be specified as an angle. Designing a BCRS with a large aperture to accommodate large FOVs conflicts with the requirement for a small cavity opening to achieve a high cavity emissivity.

Some RTs, particularly low-cost models, have large FOVs which call for very large cavity apertures. Fig. 1 shows a selection of FOVs, including FOVs commonly available on such low-cost RT models. In addition, the FOV always extends beyond the nominal spot size due to the Size-of-Source Effect (SSE), and thus a still larger source must be used so the RT receives the full signal $[8,9]$. Target areas double or triple the nominal spot size have been suggested as rules-of-thumb to circumvent the SSE $[10,11]$. The RT may be brought closer to the BCRS in order to fill its FOV, but this can present problems at extreme temperatures if convective heat transfer from the source raises or lowers the temperature of the RT's detector excessively.

A further design consideration is whether the RT will be positioned with its focal plane at the entrance to the cavity or on
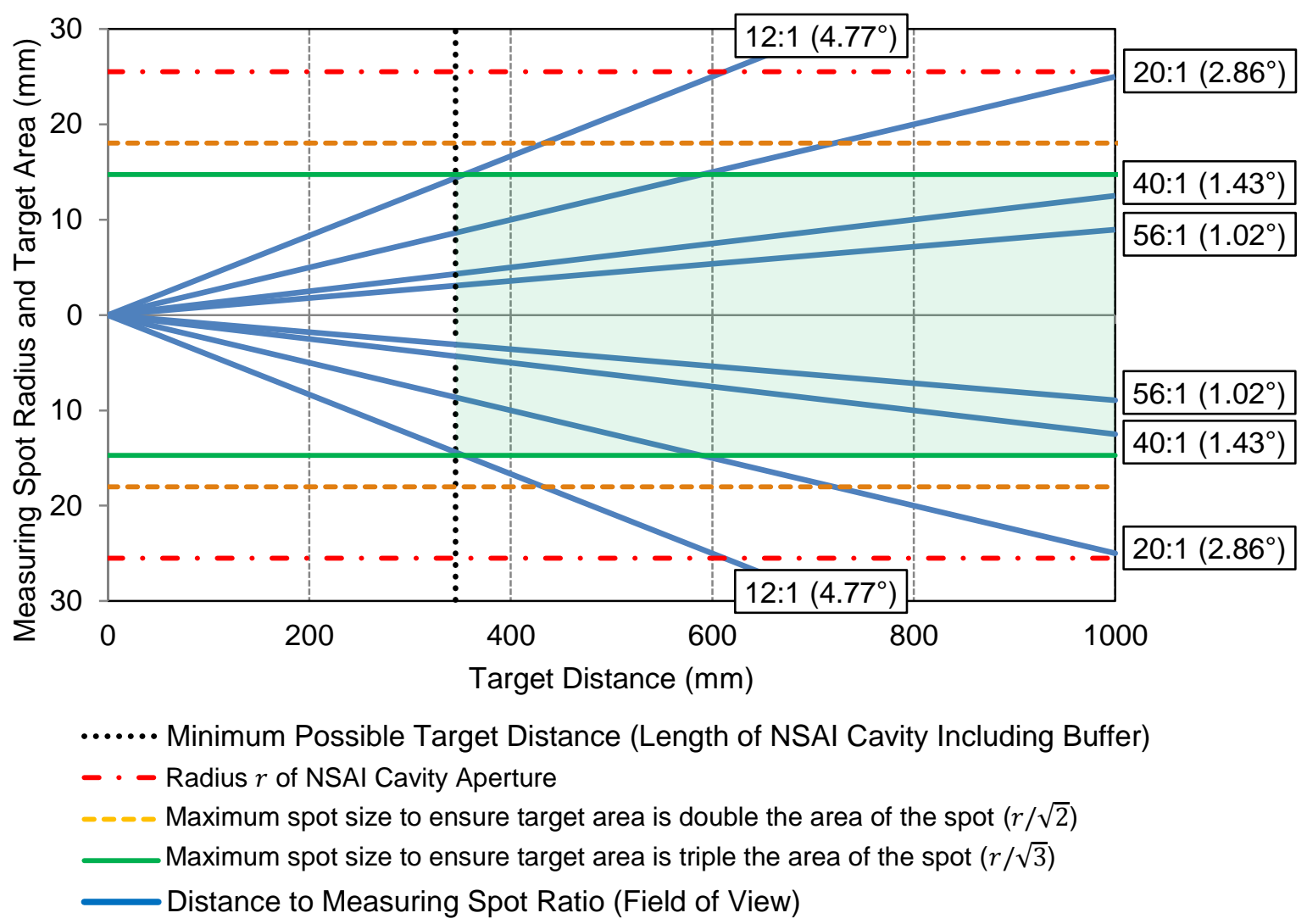

Fig. 1. Target distances and nominal spot sizes, with a selection of commonly specified fields of view (not to scale). For illustrative purposes, the FOVs are shown with the RT lenses positioned at the origin or cavity aperture and the distance to the base of the NSAI BCRS is shown. The shaded area indicates the acceptable target distances, which may be achieved by moving the RT back away from the aperture, when measuring the NSAI BCRS. The FOV of the TRT IV. 82 is also shown $(56: 1)$. 
the cavity bottom. Focussing on the cavity aperture requires that the cavity walls have a highly uniform temperature along their length - conditions best achieved using a heat-pipe BCRS. This is because the entirety of the cavity's interior is measured and the effective emissivity of the cavity in its totality is measured. The smallest errors for bath-based cavities are often obtained when the RT is focused, as above, on the cavity bottom, as this is where the FOV is filled and the effective emissivity is highest [12].

In order to reduce the specular reflectivity of the end of the cavity, a cone shape or an inclined plane is typically used. A conical end is easy to construct if the cavity is bored out and allows simplified analysis of the emissivity, but an inclined plane is easier to construct with thin walls or using sheet materials.

\subsection{The NSAI BCRS}

The NSAI cavity discussed in this paper is shown in Fig. 2. The vertical NSAI BCRS was formed from commercially available copper pipe of length $(l) 350 \mathrm{~mm}$, with internal diameter $(d)$ $51 \mathrm{~mm}$ and wall thickness $1.3 \mathrm{~mm}$. The cavity bottom was cut at an angle and sealed with a flat section of copper sheet.

Fig. 1 shows common RT FOV specifications which may be calibrated with a BCRS of these dimensions. RTs with FOVs of less than $4.77^{\circ}$, falling within the shaded area of the graph, may be calibrated with this cavity. RTs with such large FOVs would, however, need to be positioned directly at the cavity aperture to ensure the FOV is filled. RTs with larger FOVs cannot be calibrated with this BCRS. Due to difficulties associated with calibrating RTs directly at the aperture, the NSAI BCRS is recommended for calibration of RTs with FOVs of approximately $2.86^{\circ}$ or less.

A high emissivity on the surface of the internal walls can be achieved by using special coatings developed for this purpose. In this temperature range, Pyromark 1200 has been shown to provide a high emissivity that is robust enough to withstand the conditions of use [13]. The walls of the cavity have been sandblasted and coated with Pyromark 1200 paint on the interior. After the last painting, the paint was dried at $100{ }^{\circ} \mathrm{C}$ for more than 24 hours.

In order to increase the immersion of the cavity in the bath medium, a polytetrafluorethylene (PTFE) spacer is used in a similar approach to that in [14]. The upper range limit was decided in part due to the PTFE spacer, which was found to swell slightly at temperatures above $150{ }^{\circ} \mathrm{C}$. (a)
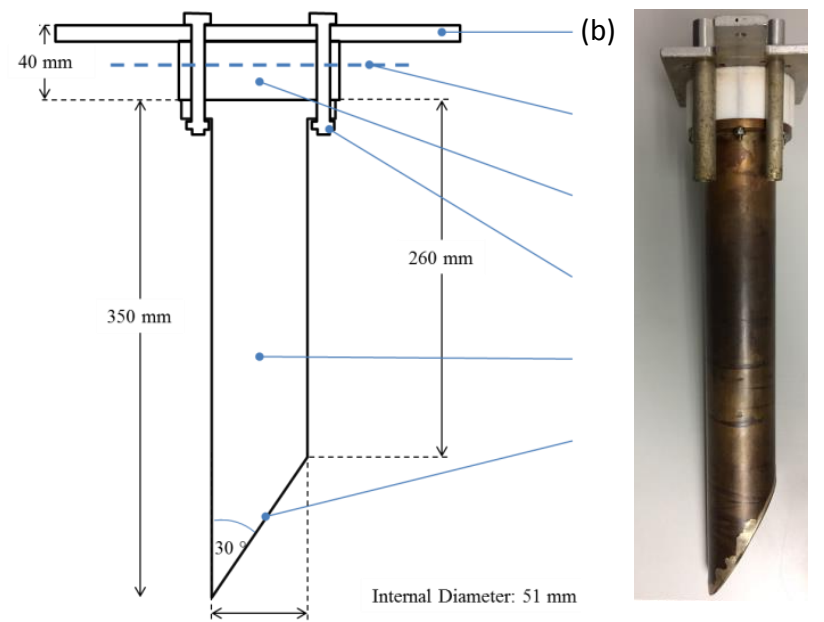

Fig. 2. The NSAI vertical BCRS (a) and (b), showing the top mounting plate and aperture (1); immersion depth (2); PTFE buffer (3); retaining bolts connecting the mounting plate, buffer and copper flange (4); copper pipe, the interior of which is coated with high emissivity paint (5) and target area (6)

\subsection{Description of the Comparison Cavities}

The details of the baths and cavities used for the comparison are given in Table 1. Including the NSAI BCRS, two vertical cavities and five horizontal cavities were compared in terms of their radiance temperature. The vertical cavities were cylindrical with $30^{\circ}$ inclined bottoms.

The LMK cavities were designed to cover the low temperature range from $-30{ }^{\circ} \mathrm{C}$ to $150{ }^{\circ} \mathrm{C}$. The horizontal cavities were cylindrical with conical bottoms of angle $120^{\circ}$. All cavities were painted with high emissivity paints and all were made of copper, apart from the LMK vertical cavity which was made of aluminium. Besides its aluminium construction, the LMK vertical BCRS differs from the NSAl cavity in that it is slightly smaller and lacks the upper mounting plate and PTFE buffer shown in Fig. 2. The LMK vertical cavity is normally mounted with the upper part of the aluminium walls projecting $30 \mathrm{~mm}$ to $40 \mathrm{~mm}$ above the liquid level of the bath to prevent the ingress of liquid. Both vertical cavities had the same shape and equal radii to within $1.5 \mathrm{~mm}$.

The TRT manufacturer used two commercially available blackbody cavities (Heitronics SW20 and SW10C) to perform the initial calibration of the TRT. The cavity sources were set to traceable temperature set points and allowed to stabilise. Radiance temperature readings were obtained from the TRT. A calibration certificate was produced and supplied with the TRT to NSAI. The calibration measurements are traceable via another TRT of the same model (TRT IV.82) to Physikalisch-Technische Bundesanstalt (PTB), the German NMI. These calibration measurements were also used in the comparison.

\subsection{Comparison Method}

Comparisons were undertaken firstly in LMK and later in NSAI. As with a regular RT calibration, the TRT was pointed at the BCRSs and the RT reading $t_{T R T}$ and the traceable contact temperature $t_{90}$ of the cavity bottom were recorded. TRT corrections were calculated as $t_{\text {corr. }}=t_{90}-t_{T R T}$.

The measurements in LMK were made with a single TRT measuring a variety of BCRSs in-house over the course of a week. The measurements in NSAI were made with a different TRT over the course of two months, starting one month after the TRT's initial calibration by the manufacturer.

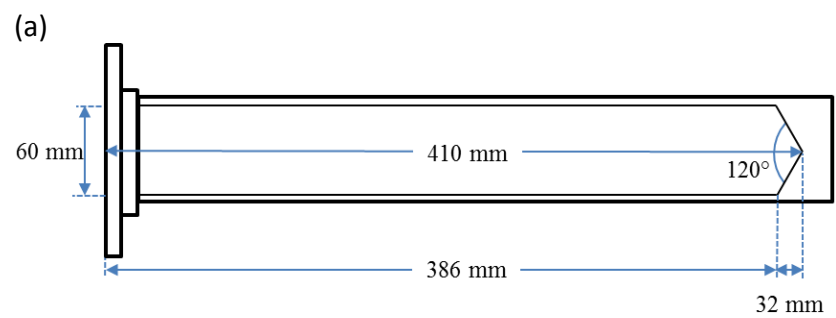

(b)

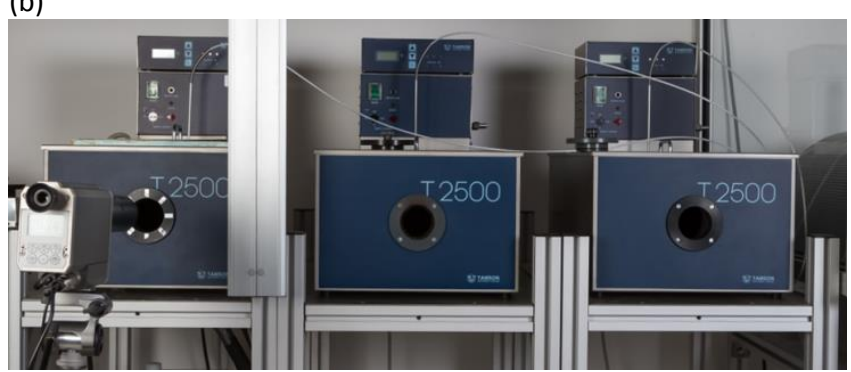

Fig. 3. Design of the LMK horizontal cavity (a) and photo showing the suite of BCRS, with the transfer radiation thermometer in the foreground on the left (b). 
Table 1

Properties of the various blackbody cavities compared, including the length $l$, diameter $d$ and the angle at the cavity's end $\theta_{\text {tip. }}$.

\begin{tabular}{|c|c|c|c|c|c|c|c|c|c|}
\hline BCRS Name & $I(\mathrm{~mm})$ & $d(\mathrm{~mm})$ & Shape & & & $\theta_{t i p}$ & $\begin{array}{l}\text { Estimated } \\
\varepsilon_{\text {cavity }}\end{array}$ & Bath Medium & Range $\left({ }^{\circ} \mathrm{C}\right)$ \\
\hline NSAI Vertical & 350 & 51 & $\begin{array}{l}\text { Cylindrical with } \\
\text { Bottom }\end{array}$ & Inclined & Flat & $30^{\circ}$ & 0.998 & $\begin{array}{l}\text { Methanol, } \\
\text { Deionised Water, } \\
\text { Mineral Oil }\end{array}$ & $\begin{array}{l}-30 \text { to } 5, \\
5 \text { to } 80 \\
80 \text { to } 150\end{array}$ \\
\hline LMK Horizontal & 450 & 60 & Cylindro-conical & & & $120^{\circ}$ & 0.999 & Ethanol water mix & -30 to 5 \\
\hline LMK Horizontal & 450 & 60 & Cylindro-conical & & & $120^{\circ}$ & 0.999 & Deionised Water & 5 to 60 \\
\hline LMK Horizontal & 450 & 60 & Cylindro-conical & & & $120^{\circ}$ & 0.999 & Mineral Oil & 60 to 150 \\
\hline LMK Vertical & 310 & 48 & $\begin{array}{l}\text { Cylindrical with } \\
\text { Bottom }\end{array}$ & Inclined & Flat & $30^{\circ}$ & 0.998 & $\begin{array}{l}\text { Methanol, } \\
\text { Deionised Water, } \\
\text { Mineral Oil }\end{array}$ & $\begin{array}{l}-30 \text { to } 20 \\
5 \text { to } 80 \\
50 \text { to } 150\end{array}$ \\
\hline SW20 Horizontal & 120 & 40 & Cylindro-conical & & & $120^{\circ}$ & $\geq 0.995$ & Deionised Water & 30 to 95 \\
\hline SW10C Horizontal & 105 & 25 & Cylindro-conical & & & $120^{\circ}$ & $\geq 0.99$ & Not bath-based & 50 to 400 \\
\hline
\end{tabular}

For each of the measurements undertaken in LMK and NSAI, the RT was manually aligned with the axis of the BCRS using a variety of mounts to replicate practical usage conditions. The RT was positioned such that its lens was $35 \mathrm{~mm}$ from the aperture of the BCRS. As such, for measurements of the NSAI BCRS, the RT was focused on centre of the cavity bottom. For measurements of the LMK horizontal BCRSs, the RT's focal plane was midway along the cavity, $65 \mathrm{~mm}$ from the bottom. Measurements were made with the cavity diameters given in Table 1 as the defining apertures and cooled apertures were not used.

The measurements in the Heitronics laboratory were made with the RT focused on the BCRS aperture, and $36 \mathrm{~mm}$ and $24 \mathrm{~mm}$ aperture plates were used with the SW20 and SW10C BCRSs respectively.

\subsection{Comparison Thermometers}

The following comparison standards were used:

- The TRT II was used for measurements in LMK. The applicable temperature range of this RT is from $-50{ }^{\circ} \mathrm{C}$ to $300{ }^{\circ} \mathrm{C}$ with spectral sensitivity from $8 \mu \mathrm{m}$ to $14 \mu \mathrm{m}$. The pyrometer has fixed-focus optics with focal length $380 \mathrm{~mm}$ and nominal spot size $6.8 \mathrm{~mm}$. The SSE characteristic of the RT was measured by the manufacturer using the direct method. At a target distance of $380 \mathrm{~mm}$, where a $10 \mathrm{~mm}$ diameter target size was used, $99 \%$ of the signal was obtained relative to that received from a $50 \mathrm{~mm}$ diameter target.

- The TRT IV.82 was used for measurements in NSAI. This RT has a single temperature range from $-50{ }^{\circ} \mathrm{C}$ to $1000{ }^{\circ} \mathrm{C}$ and spectral sensitivity from $8 \mu \mathrm{m}$ to $14 \mu \mathrm{m}$. The optical characteristics are identical to those of the TRT II described above.

- A selection of calibrated SPRTs and bridges were used to make contact thermometry measurements that were traceable to ITS-90.

\subsection{Vertical Cavity Measurements}

In order to carry out non-contact temperature measurements of the vertical cavities, they were each immersed in turn in the baths and fixed in place.

The baths were allowed to stabilise for approximately 2 hours at each of the set-points throughout the temperature range. The stability of the baths was better than $\pm 0.01{ }^{\circ} \mathrm{C}$ at each set point.
The bath temperature was monitored using SPRTs that were immersed beside the cavities such that the sensing element was within $40 \mathrm{~mm}$ of the target area at the cavity bottom. Secondary contact thermometer references were also used as crosschecks.

The cavities were then measured with a TRT. The optical axis of the RT was aligned with the axis of the cavity and the RT was positioned so that it was focused on the centre of the cavity bottom. Each measurement round consisted of ten measurements of the contact temperature and non-contact temperature references, recorded at 10 second intervals using data acquisition software.

To minimise the formation of condensation and ice at temperatures below ambient, a lid was used. The lid was kept in place when the bath was stabilising and removed prior to the TRT measurements. The exterior walls of the vertical cavities were cleaned and dried after use in each media.

\subsection{Horizontal Cavity Measurements at LMK}

The LMK horizontal cavities are all permanently sealed onto the sides of dedicated baths. The contact temperature was again monitored using SPRTs. The TRT was aligned manually in front of the cavity aperture, in a similar manner to that for the vertical measurements described in section 2.6.

The low temperature LMK horizontal cavity is equipped with a gas purging system to prevent ice formation on the cavity walls at temperatures below ambient. Purging was carried using nitrogen gas of $5 \mathrm{~N}$ purity at flow rates of approximately 1 litre per minute.

\section{Comparison Results}

\subsection{Comparison of the NSAI Vertical Cavity with Horizontal Cavities in $L M K$}

Fig. 4 shows the comparison measurements made between the NSAI vertical BCRS and the suite of horizontal cavities in LMK. The horizontal cavities were chosen according to the temperature ranges shown in Table 1 . The uncertainty of measurement was estimated based on the model suggested by the CCT-WG5 [15]. The difference between the magnitude of the TRT corrections for the vertical and horizontal cavities $\left|t_{\text {corr. vertical }}\right|-\left|t_{\text {corr. horizontal }}\right|$ was less than $0.25{ }^{\circ} \mathrm{C}$ across the temperature range. 


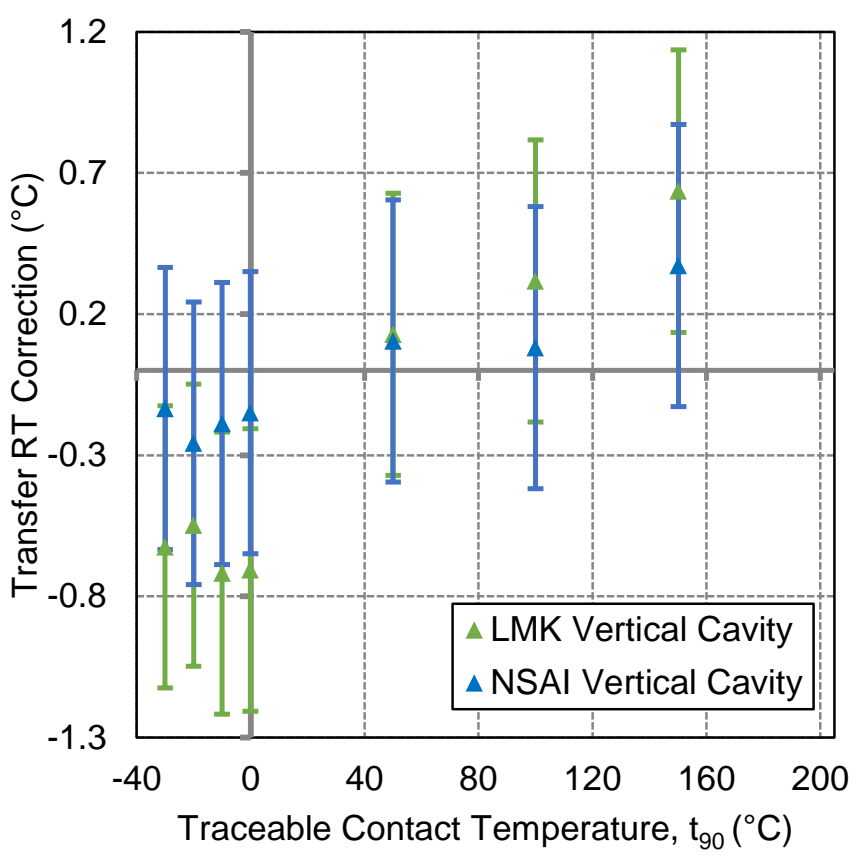

Fig. 4. Comparison of the NSAI vertical BCRS and the vertical LMK BCRSs in the range $-30{ }^{\circ} \mathrm{C}$ to $150{ }^{\circ} \mathrm{C}$. The error bars show the estimated uncertainty $(k=2)$.

\subsection{Comparison of the NSAI vertical cavity with vertical cavity in $L M K$}

In addition to comparing the NSAI vertical cavity to horizontal cavities, it was compared to a similar vertical cavity that had previously been constructed at LMK. Both of these cavities were removable and so they could be immersed simultaneously in the same baths for the measurement experiments at the temperatures from $50{ }^{\circ} \mathrm{C}$ to $150{ }^{\circ} \mathrm{C}$. This comparison was conducted to investigate the influences of the design differences, described in section 2.3 , of the two cavities.

The results of the comparison are shown in Fig. 4. The corrections for the NSAI cavity were smaller than the LMK vertical cavity at each set point.

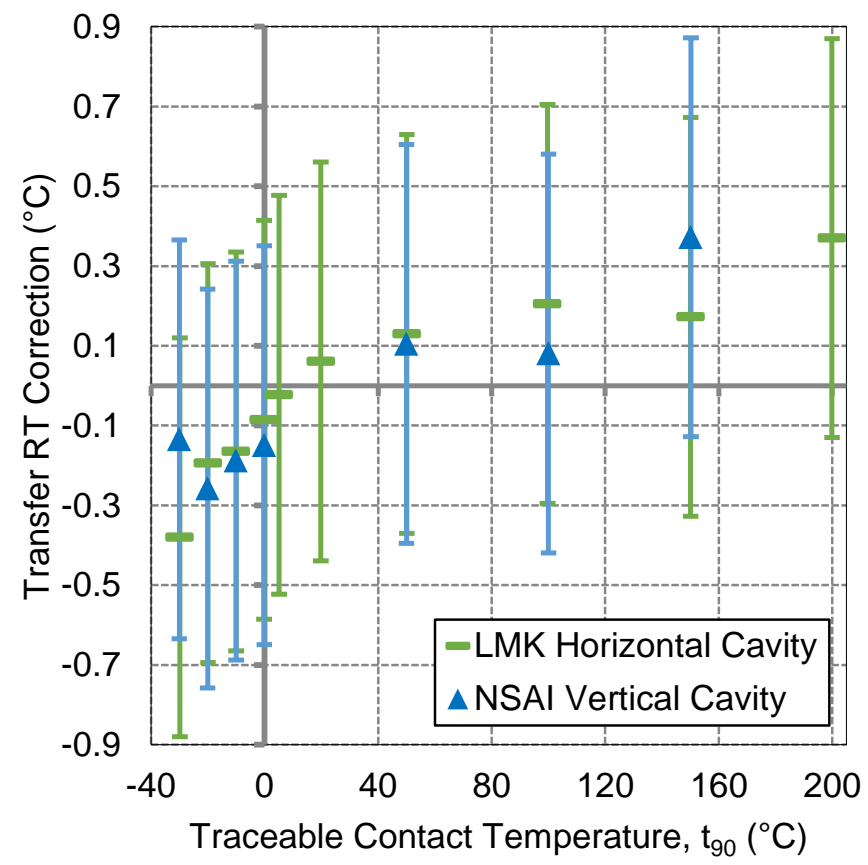

Fig. 5. Comparison of the NSAI vertical BCRS and the horizontal LMK BCRSs in the range $-30{ }^{\circ} \mathrm{C}$ to $150{ }^{\circ} \mathrm{C}$. The error bars show the estimated uncertainty $(k=2)$.

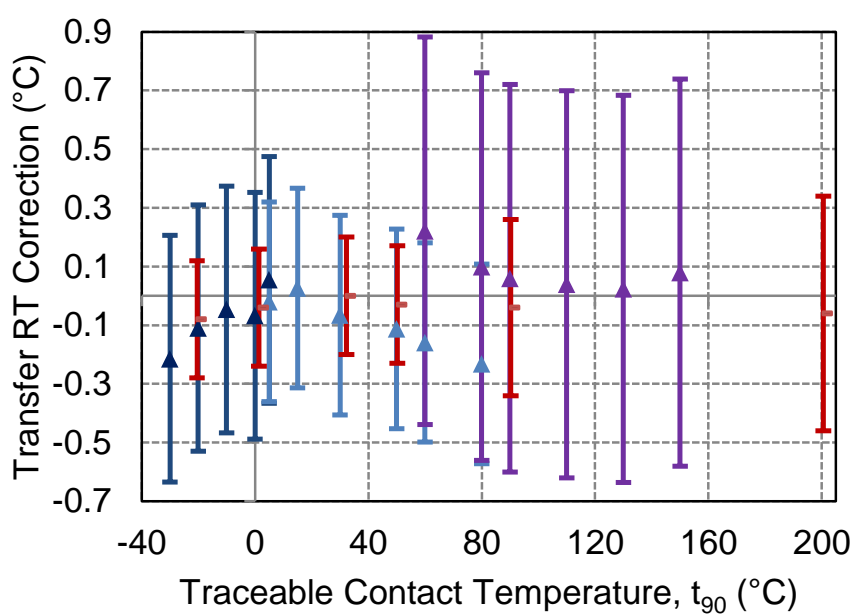

\section{$\Delta$ Methanol Bath \\ $\triangle$ Water Bath \\ $\triangle$ Oil Bath \\ - Manuf. Calibration (Horizontal), Traceable to PTB}

Fig. 6. Comparison of measurements made by the RT manufacturer of horizontal BCRSs with measurements made of the NSAl vertical BCRS. The error bars show the estimated uncertainty $(k=2)$.

\subsection{Comparison of the NSAI Vertical Cavity against TRT Manufacturer's Measurements}

Following the work conducted in LMK, a further comparison was made possible by NSAl's procurement of an in-house TRT. Measurements made in NSAl were compared to recent TRT calibration results received from the manufacturer.

The measurements made in NSAI were made in much the same way as those for the vertical comparisons at LMK, using upwelling baths, and traceable SPRTs and bridges. Two changes to the measurement procedure were made:

- A larger top plate was used to further reduce the possibility of the bath liquid entering the cavity and limit the influence of bath vapours on the TRT lens;

- A manually-positioned stand to mount the TRT vertically above the BCRS was used.

A summary of the uncertainty components used for the measurements made in NSAI is given in Table 2. The simplified budget follows the structure used in the CCT-WG5/08-03 document [15], with the exception of the contributions due to blackbody emissivity and repeatability, where aggregate components are used. The blackbody emissivity component comprises the isothermal and non-isothermal emissivity contributions. Values for the other contributions which were not measured are the sourced from CCT-WG5/08-03 and ASTM E2847 [16] with the most conservative values being used.

The uncertainty was evaluated in order to give a conservative estimate. The most conservative value for each of the measured components was used across the range of a given bath media. In the case of the contact measurement and cavity bottom uniformity components, the most conservative estimate was applied across the entire temperature range. 
Table 2

Uncertainty values for the NSAI BCRS measured with the TRT IV.82 over the three ranges of thermal medium.

\begin{tabular}{|c|c|c|c|}
\hline Source of uncertainty & $\begin{array}{c}\text { Methanol } \\
-30{ }^{\circ} \mathrm{C} \text { to } \\
5{ }^{\circ} \mathrm{C} \\
\pm \mathrm{mK}\end{array}$ & $\begin{array}{l}\text { Water } \\
5{ }^{\circ} \mathrm{C} \text { to } \\
80{ }^{\circ} \mathrm{C} \\
\pm \mathrm{mK}\end{array}$ & $\begin{array}{c}\text { Oil } \\
80^{\circ} \mathrm{C} \text { to } \\
150{ }^{\circ} \mathrm{C} \\
\pm \mathrm{mK}\end{array}$ \\
\hline \multicolumn{4}{|l|}{ BCRS Components } \\
\hline $\begin{array}{l}\text { NML Contact Uncertainty } \\
\text { of Measurement }^{+}\end{array}$ & 13 & 13 & 13 \\
\hline $\begin{array}{l}\text { Blackbody Emissivity } \\
\text { Reflected Ambient } \\
\text { Radiation }\end{array}$ & 99 & 49 & 29 \\
\hline $\begin{array}{l}\text { Cavity Bottom Heat } \\
\text { Exchange }\end{array}$ & 0 & 9 & 9 \\
\hline Convection Heat Loss & 17 & 17 & 33 \\
\hline Cavity Bottom Uniformity & 63 & 63 & 63 \\
\hline $\begin{array}{l}\text { Blackbody Ambient } \\
\text { Conditions }\end{array}$ & 58 & 58 & 58 \\
\hline \multicolumn{4}{|l|}{ RT Components } \\
\hline Size-of-Source Effect & 25 & 25 & 25 \\
\hline Reference Temperature & 100 & 100 & 100 \\
\hline Ambient Temperature & 51 & 51 & 51 \\
\hline Atmospheric Absorption * & 33 & 33 & 59 \\
\hline Repeatability $^{\dagger}$ & 95 & 44 & 275 \\
\hline Readout Resolution & 29 & 29 & 29 \\
\hline \multicolumn{4}{|l|}{ Combined Standard } \\
\hline Uncertainty & 209 & 171 & 330 \\
\hline $\begin{array}{l}\text { Expanded Uncertainty } \\
(k=2)\end{array}$ & 418 & 342 & 660 \\
\hline \multicolumn{4}{|c|}{${ }^{+}$indicates values based on measurements in NSAI } \\
\hline
\end{tabular}

\section{Discussion}

Overall, the results show agreement between the horizontal and vertical configurations in this temperature range.

Fig. 4 shows good agreement between the vertically and horizontally configured BCRSs at each of the temperature set points. The magnitude of the TRT corrections for the vertical cavity is larger at most but not all set points. The figure indicates that the corrections for the vertical cavity increase at the top end of the temperature range. This could be indicative of an upper limit for this set-up. Use of a vertical cavity may cause problems in temperatures above the range of the comparison due to the effects of convection on the measuring RT.

Some ice did form on the walls of the vertical cavities near the aperture at temperatures below zero for the measurement experiments performed in LMK and NSAI. Ice formation near the target area, deeper within the cavity, however, was observed to be minimal. The TRT correction was usually negative below $0{ }^{\circ} \mathrm{C}$. Fig. 4 shows that the TRT corrections for the NSAI vertical cavity were smaller than those for the LMK vertical cavity, indicating an improvement in performance. Further work is needed to determine which of the design differences described in section 2.3 had an influence on the performance improvement, which was up to $0.56{ }^{\circ} \mathrm{C}$. The NSAl vertical cavity benefited from an increased length, a design that improved immersion and therefore uniformity, a top plate around the aperture and a copper rather than aluminium construction. These are some potential reasons for the improved performance.

It should be noted that in each of the comparison figures 4, 5 and 6 , any intrinsic influence on the measurements due to the TRT is not known. A relative comparison of the TRT corrections for each cavity in Fig. 4 and Fig. $\mathbf{4 5}$ gives an indication of this influence. The effectiveness of the PTFE collar shown in Fig. 2 could be improved by redesigning bolting configuration so that bolts mounting alternately from the top plate and the copper flange are used, thus eliminating heat bridges as in [14]

\section{Conclusions}

In summary, a vertical BCRS was designed, constructed, and evaluated. The cavity was found to offer a flexible, low-cost alternative to purpose-built horizontal cavities. The new design was also found to be an improvement over the previous vertical cavity constructed at LMK. It is expected that such vertical cavities could be of use to other calibration laboratories wishing to extend the temperature range of their non-contact capabilities. A vertical orientation may also assist in the assessment of prototype cavities with different dimensions or provide additional cavities to reduce turnaround times, or for crosschecks.

The evaluated expanded uncertainties $(k=2)$ ranged from $0.66{ }^{\circ} \mathrm{C}$ when the cavity was immersed in the oil bath to $0.34{ }^{\circ} \mathrm{C}$ when immersed in the water bath. The key outcome is that the use of vertically oriented cavities is a valid method for achieving repeatable measurement traceable to ITS-90.

\section{Acknowledgements}

This work was supported by the European Metrology Programme for Innovation and Research through the Eura-Thermal project and by NSAI. Sam Boles gratefully acknowledges the staff of both LMK and the National Physical Laboratory (UK) for their help and hospitality during measurement and training visits. Sam Boles also acknowledges the assistance of Brian Cusack prior to and at the commencement of this work and the countless contributions of his supervisors and other staff in DIT and NSAI.

\section{References}

[1] D.P. DeWitt, G.D. Nutter, Theory and Practice of Radiation Thermometry, 1st ed., Wiley, 1988. doi:10.1002/9780470172575.

[2] J. Hartmann, High-temperature measurement techniques for the application in photometry, radiometry and thermometry, Phys. Rep. 469 (2009) 205-269. doi:10.1016/j.physrep.2008.09.001.

[3] K.D. Hill, The NRC Blackbody-Based Radiation Thermometer Calibration Facility, AIP Conf. Proc. 684 (2003) 669674. doi:10.1063/1.1627204.

[4] P.R. Dekker, E. Van Der Ham, ITS-90 scale realization on the new radiation thermometer calibration facility at NMi VSL, Int. J. Thermophys. 29 (2008) 1001-1013. doi:10.1007/s10765-0080420-2.

[5] R. Simpson, G. Machin, H. McEvoy, R. Rusby, Traceability and calibration in temperature measurement: a clinical necessity, J. Med. Eng. Technol. 30 (2006) 212-7. doi:10.1080/03091900600711530.

[6] J.V. Nicholas, D.R. White, Traceable Temperatures, 2nd ed., John Wiley \& Sons Ltd, 2001.

[7] P. Saunders, MSL Technical Guide 22 Calibration of Infrared Thermometers, 64 (2009) 1-8.

[8] Y. Yamada, J. Ishii, Toward Reliable Industrial Radiation Thermometry, Int. J. Thermophys. (2015) 1699-1712. doi:10.1007/s10765-015-1870-y.

[9] I. Pušnik, G. Grgić, J. Drnovšek, Calculated uncertainty of temperature due to the size-of-source effect in commercial radiation thermometers, Int. J. Thermophys. 29 (2008) 322-329. doi:10.1007/s10765-007-0330-8 
[10] I. Pušnik, G. Grgić, J. Drnovšek, System for the determination of the size-of-source effect of radiation thermometers with the direct reading of temperature, Meas. Sci. Technol. 17 (2006) 1330-1336. doi:10.1088/0957-0233/17/6/007.

[11] F. Liebmann, Infrared Thermometer Calibration, Cal Lab Int. J. Metrol. (2011) 20-22. doi:10.1126/science.189.4201.483.

[12] R.E. Bedford, I. Introduction, Design of an isothermal cavity with nonuniform local instrinsic emissivities to give true blackbody radiant characteristics, (1992) 3213-3216.

[13] X. Song, K. Huan, W. Dong, J. Wang, Y. Zang, X. Shi, Research on infrared radiation characteristics of Pyromark 1200 high-temperature coating, in: M. Guina, H. Gong, Z. Niu, J. Lu (Eds.), Int. Symp. Optoelectron. Technol. Appl. 2014, International Society for Optics and Photonics, 2014: p. 93001 S. doi:10.1117/12.2072577.

[14] EN 12470-5: Clinical thermometers - Part 5: Performance of infra-red ear thermometers (with maximum device), (2003).

[15] P. Saunders, J. Fischer, M. Sadli, M. Battuello, C.W. Park, Z. Yuan, H.W. Yoon, W. Li, E. Van Der Ham, F. Sakuma, J. Ishii, M. Ballico, G. Machin, N. Fox, J. Hollandt, M. Matveyev, P. Bloembergen, S. Ugur, CCT-WG5/08-03 Uncertainty budgets for calibration of radiation thermometers below the silver point, Paris, 2008. doi:10.1007/s10765-008-0385-1.

[16] ASTM E2847 - 14 Standard Test Method for Calibration and Accuracy Verification of Wideband Infrared Thermometers, $i$ (2014) 1-17. doi:10.1520/E2847. 